\title{
Safety of Diagnostic Ultrasound No. 6. The Safety of Pulsed Doppler Ultrasound
}

\section{Kazuo Maeda*}

Department of Obstetrics and Gynecology (Emeritus), Tottori University Medical School, Yonago, Japan

\begin{abstract}
There are two pulsed Doppler ultrasound including continuous wave (CW) Doppler and pulsed Doppler, where the safety is discussed in the pulsed Doppler, because of its higher ultrasound intensity, due to longer pulse and higher pulse repetition frequency than simple B-mode ultrasound, and obstetrical setting of ultrasound devices determines the thermal and mechanical indices below one in pulsed Doppler. Ultrasound user is responsible to ultrasound safety, where the user lowers the ultrasound thermal and mechanical indices below one, when indices are higher than one. The use of pulsed Doppler in the first trimester is regulated by ISUOG committee.
\end{abstract}

Keywords: Ultrasound;Pulsed doppler;Bioeffet; TI; MI;First trimest er;Ductusvenosus;Chromosomal abnormality

\section{Two Kinds of Doppler Ultrasound}

There are continuous wave (CW) Doppler and pulsed Doppler in the Doppler ultrasound. Although there is no safety problem in CW Doppler, because of very low ultrasound intensity in CW Doppler, big advantage of pulsed Doppler is to identify the analyzing location by the sample volume, while it is unable in CW Doppler, despite ultrasound safety is discussed in pulsed Doppler, because ultrasound intensity tends to be high in pulsed Doppler due to wide ultrasound pulse and high pulse repetition frequency.

\section{The Recording of Pulsed Doppler Flow Wave}

An ultrasound pulse, which is longer than B-mode device, is generated in a ultrasound unit in the probe, and an arterial pulsating blood flow is exposed to the ultrasound pulse at the sampling volume of pulsed Doppler ultrasound beam (Figures 1-3).

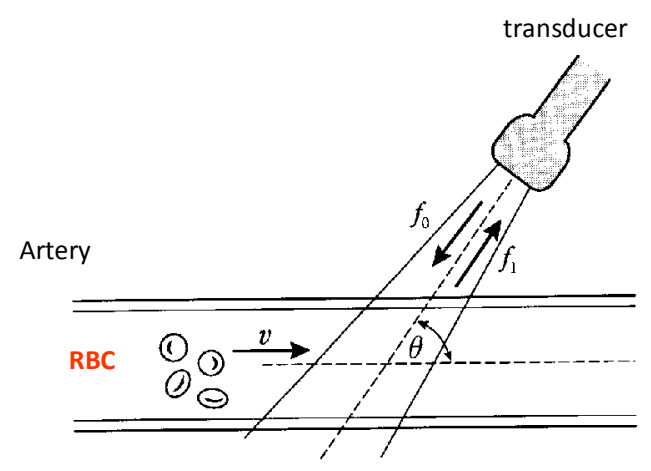

$\begin{aligned} & \text { Doppler } \\ & \text { frequency }\end{aligned} f_{d}=f_{1}-f_{0}=\left(2 v f_{0} \cos \theta\right) / c$

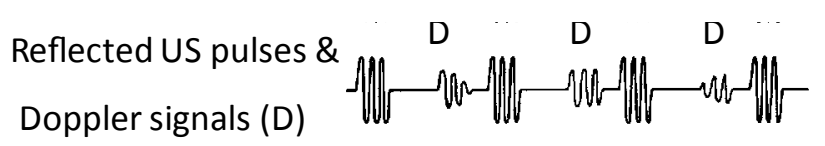

Figure 1: Formation of Doppler blood flow signal.

Doppler signal $(D)=f 1-f 0=(2 v f 0 \cos \theta) / c$

$\mathrm{V}$ : Red blood cell flow velocity, c: f0 ultrasound propagation speed

\section{Intensity of Doppler Ultrasound}

Pulsed Doppler signal develops according to the subject velocity shown by the frequency of Doppler signal. In pulsed Doppler ultrasound, pulse wave is wider, pulse repetition frequency (PRF) is higher than B-mode, then pulsed Dopplerultrasound intensity tends higher than simple B-mode to clearly image the flow velocity curves. The PRF and ultrasound intensity are able to reduce by the user to keep TI and MI of pulsed Doppler ultrasound less than 1.0, when safety indices (TI or MI) are higher than 1.0 on the monitor display.

\section{Doppler signals in a few $\mathrm{mS}$.}
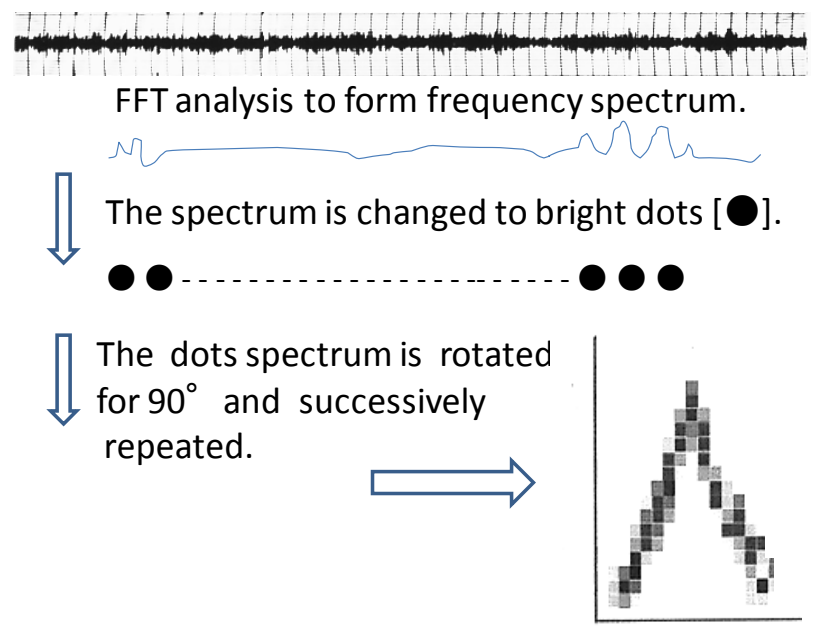

Figure 2: Formation of pulsed Doppler flow velocity wave.

*Corresponding author: Kazuo Maeda, Department of Obstetrics and Gynecology (Emeritus), Tottori University Medical School, 3-155, Nadamachi, Yonago, 683-0835 Japan, Tel: 81-859-22-6856; Fax: 81-859-22-6856; E-mail: maedak@mocha.ocn.ne.jp

Received March 19, 2014; Accepted May 15, 2014; Published May 19, 2014

Citation: Maeda K (2014) Safety of Diagnostic Ultrasound No. 6. The Safety of Pulsed Doppler Ultrasound. J Health Med Informat 5: 155. doi:10.4172/21577420.1000155

Copyright: ( 2014 Maeda K. This is an open-access article distributed under the terms of the Creative Commons Attribution License, which permits unrestricted use, distribution, and reproduction in any medium, provided the original author and source are credited. 


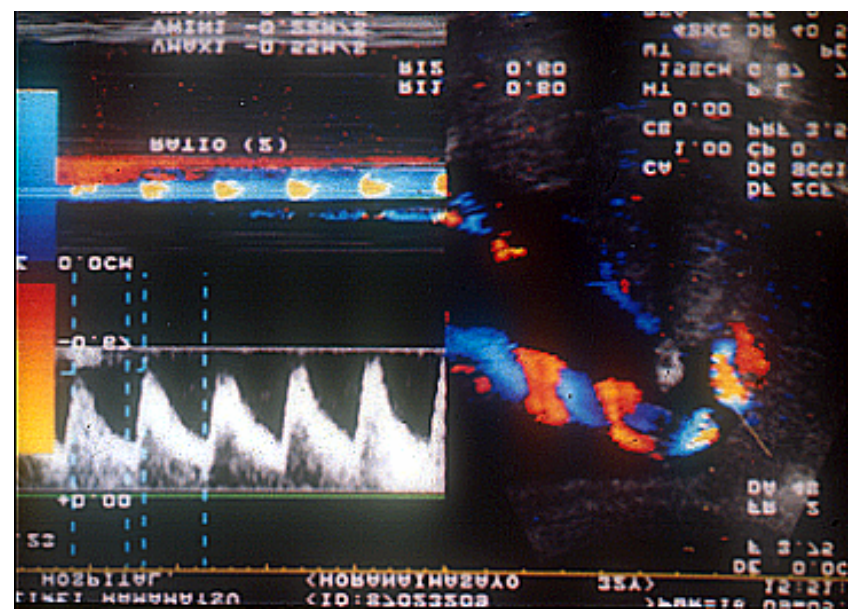

Figure 3: Pulsed Doppler umbilical arterial flow velocity wave and color Doppler flow mapping of umbilical arterial and vein blood flows.

\section{Comparison to B-mode}

No hazardous thermal effect is expected in simple B-mode imaging device because of minimum heat production due to low ultrasound average intensity, i.e. World Federation of Ultrasound in Medicine and Biology (WFUMB) [1] concluded that the use of simple imaging equipment is not contraindicated on thermal grounds. The real-time B-mode, simple three dimensional (3D) and four dimensional (4D) imaging devices are included in that category. However, too long exposure to simple real-time B-mode ultrasound, e.g. longer than 30 min, is avoided, because of the disturbance of neuronal cell migration in fetal animal brain by the longer exposure than $30 \mathrm{~min} \mathrm{[2].}$

\section{Regulation of Pulsed Doppler Ultrasound}

Diagnostic ultrasound safety was perfect in Japan, after the regulation of Japanese Industrial Standard on the output power of diagnostic ultrasound devices below $10 \mathrm{~mW} / \mathrm{cm}^{2}$ in 1980 , where the level was $1 / 100$ of hazardous $1.0 \mathrm{w} / \mathrm{cm}^{2}$ of CW ultrasound and $1 / 24$ of hazardous $240 \mathrm{~mW} / \mathrm{cm}^{2}$ of PW[3-5]. However, ultrasound safety has been discussed again after the introduction of pulsed Doppler, because pulsed Doppler method intensity tends to be higher than simple B-mode ultrasound, as listed above. An ultrasound user should be prudent, when the pulsed Doppler is accompanied by simple B-mode devices, e.g. 3D and 4D ultrasound. That is the reason why TI and MI are less than 1.0 in obstetrical setting for the fetal study.

\section{Thermal and Mechanical Indices}

The thermal effect is big concern in Doppler ultrasound study. Tissue temperature rises not only at the sample volume, but also in all tissues passed by the pulsed Doppler ultrasound beam. The International Society of Ultrasound in Obstetrics and Gynecology (ISUOG) discussed the safe use of Doppler ultrasound [6]. Ultrasound intensity is less in color/power Doppler flow mapping due to its scanning procedure than stable irradiation of pulsed Doppler to fixed point.

The MI also regulated below 1.0, because the main mechanical ultrasound bioeffect is the cavitation, which accompanies high pressure, high temperature and free radical formation, will not occur in the cell plasma of high viscosity and no gas bubble, and free radical formed in the liquid does not reach the cell due to its short life span, but high intensity ultrasound developed hemorrhage in neonatal animal lung, the $\mathrm{MI}$ is regulated less than 1.0 in obstetrical setting.

In general, it is emphasized that ultrasonic examination should be performed only by medical indications, because diagnostic ultrasound users should recognize the sensitivity of young biological tissues of developing embryos and fetuses to intense ultrasound. The users also should know ultrasonic intensity of their devices, the mechanisms of ultrasound bioeffect, and the prudent use of the devices, because the ultrasound user is responsible to the ultrasound safety [7].An important ultrasound bioeffect is thermal effect due to temperature rise induced by ultrasound absorption, because malformations were reported after the exposure of animal embryos and fetuses to high temperature in biological experiments [8]. Inertial cavitation and other mechanical effects are concerned in the non-thermal bioeffects of ultrasound. In clinical situations, the thermal and mechanical indices (TI and MI) of ultrasonic devices are less than 1.0 in obstetrical setting, and an ultrasound user reduces the output intensity of ultrasound devices to control the thermal and mechanical indices less than 1.0, when they are higher than 1.0.

Although the pulsed Doppler is useful to study the blood flow, peak flow velocity, peripheral blood flow impedance (RI and PI), and a bad fetal outcome was known in cases of the loss and reverse flow of Doppler diastole in fetal artery, the study should be prudent, if the fetal body is exposed to pulsed Doppler ultrasound due to its potential high intensity caused by the long ultrasound pulse, high repetition frequency and FFT frequency analysis of Doppler wave.

\section{Pulsed DoplerUltrasound in the First Trimester}

Although chromosomal abnormalities were detected in the reversed atrial diastole (a-wave) of fetal ductusvenosus pulsed Doppler and useful in the prenatal diagnosis of chromosomal abnormalities in the first trimester [9], a recent study on the pulsed Doppler ultrasound exposure of rat fetal ductusvenosus in early pregnancy resulted the transient increase of fetal liver apoptosis index [10], thus the ultrasound bioeffect and safety committee of ISUOG declared a statement on the use of pulsed ultrasonic Doppler ultrasound in $11-13$ weeks ${ }^{+6}$ of pregnancy in the first trimester as follows [11];

1. Pulsed Doppler (spectral, power and color flow imaging) ultrasound should not be used routinely.

2. Pulsed Doppler ultrasound may be used for clinical indications such as refine risks for trisomies.

3. When performing Doppler ultrasound, the displayed thermal index (TI) should be $=<1.0$ and exposure time should be kept as short as possible (usually no longer than $5-19 \mathrm{~min}$ ) and should not exceed $60 \mathrm{~min}$.

4. When using Doppler ultrasound for research, teaching and training purposes, the displayed TI should be $\leq 1.0$ and exposure time should be kept as short as possible (usually no longer than 5-10 $\mathrm{min}$ ) and should not exceed $60 \mathrm{~min}$. Informed consent should be obtained.

5. In educational settings, discussion of first trimester pulsed or color Doppler should be accompanied by information on safety and bioeffects (e.g. TI, exposure times and how to reduce output power).

6. When scanning maternal uterine arteries in the first trimester, there are unlikely to be any fetal safety implications as long as the embryo/fetus lies outside the Doppler ultrasound beam. 
Citation: Maeda K (2014) Safety of Diagnostic Ultrasound No. 6. The Safety of Pulsed Doppler Ultrasound. J Health Med Informat 5: 155. doi:10.4172/2157-7420.1000155

\section{Conclusion}

The use of pulsed Doppler ultrasound should be prudent in fetal study, because of its tendency to be high. The TI and MI should be lower than 1.0 in the obstetrical setting. The user should depress TI or MI below 1.0, if the monitor screen shows higher index than 1.0. The use of pulsed Doppler is regulated in the first trimester.

\section{References}

1. Barnett SB, Kossoff G (1992) WFUMB symposium on safety and standardisation in medical ultrasound: Issues and recommendations regarding thermal mechanisms for biological effects of ultrasound. Hornbick. Ultrasound Med Biol 18: 731-814.

2. Ang ES Jr, Gluncic V, Duque A, Schafer ME, Rakic P (2006) Prenatal exposure to ultrasound waves impacts neuronal migration in mice. Proc Natl Acad Sci U S A 103: 12903-12910.

3. Ide M (1986) Japanese policy and status of standardisation. Ultrasound Med Biol 12: 705-706.

4. Maeda K, Ide M (1986) The limitation of the ultrasound intensity for diagnostic devices in the Japanese industrial standards. IEEE Trans Ultrason Ferroelectr Freq Control 33: 241-243
5. Maeda K, Murao F, Yoshiga T, Yamauchi C, Tsuzaki T (1986) Experimental studies on the suppression of cultured cell growth curves after irradiation with CW and pulsed ultrasound. IEEE Trans Ultrason Ferroelectr Freq Control 33 186-193.

6. ISUOG Bioeffects and Safety Committee, Abramowicz JS, Kossoff G, Marsa K, Ter Haar G (2002) Safety statement, 2000 (reconfirmed 2002). Ultrasound Obstet Gynecol 2002 19: 105.

7. American Institute of Ultrasound in Medicine (1992) Standard for Real Time Display of Thermal and Mechanical Acoustic Output Indices on Diagnostic Ultrasound Equipment. National Electrical Manufacturers Association.

8. National Council on Radiation Protection and Measurements (1992) Exposure Criteria for Medical Diagnostic Ultrasound: I. Criteria Based on Thermal Mechanisms. NCRP Report No.113.

9. Florianski J, Fuchs T, Zimmer M, Homola W, Pomorski M, et al. (2013) The role of ductus venosus Doppler flow in the diagnosis of chromosomal abnormalities during the first trimester of pregnancy. Adv Clin Exp Med 22: 395-401.

10. Pellicer B, Herraiz S, Táboas E, Felipo V, Simon C, et al. (2011) Ultrasound bioeffects in rats: quantification of cellular damage in the fetal liver after pulsed Doppler imaging. Ultrasound Obstet Gynecol 37: 643-648.

11. Salvesen KÅ, Lees C, Abramowicz J, Brezinka C, Ter Haar G, et al. (2011) Safe use of Doppler ultrasound during the 11 to $13^{+6}$ week scan: is it possible? Ultrasound Obstet Gynecol 37: 625-628. 